\title{
El carácter dialógico-imaginativo de la creatividad y la innovación en Adam Smith
}

\section{Gonzalo Carrión*}

Universidad Nacional de Villa María gcarrion@unvm.edu.ar

\author{
Revista Cultura Económica \\ Año XXXIX • N¹01 \\ Junio 2021: 32-49 \\ https://doi.org/10.46553/cecon.39.101.2021.p32-49
}

\begin{abstract}
Resumen: El énfasis puesto por Adam Smith en el incremento de la productividad ocasionada por la división del trabajo en la Riqueza de las naciones llevó a que su concepción general sobre la dinámica del crecimiento económico se asociara casi exclusivamente con la especialización productiva del trabajo y la libre expansión los mercados competitivos. En este artículo intentaremos mostrar otra perspectiva sobre el crecimiento económico smithiano. Dicha perspectiva se centra en dos aspectos fundamentales: por un lado, la importancia de la imaginación en los procesos creativoinnovativos, y, por otro, el basamento dialógico de la división del trabajo. De esta manera, proponemos que una lectura integral de la obra smithiana resulta relevante, no solamente para alcanzar una comprensión más acabada de las propias ideas económicas del autor, sino también para brindar interesantes sugerencias para pensar la dinámica del crecimiento y desarrollo en la actualidad.
\end{abstract}

Palabras clave: Adam Smith; Crecimiento económico; Creatividad; Innovación; División del trabajo; Imaginación; Lenguaje

\section{The dialogic-imaginative character of creativity and innovation in} Adam Smith

Abstract: Adam Smith's emphasis on the increase in productivity brought about by the division of labour in the Wealth of Nations led his general conception of the dynamics of economic growth to be associated almost exclusively with the productive specialization of labour and the free expansion of competitive markets. In this article we will try to show another perspective on Smithian economic growth. This perspective focuses on two fundamental aspects: on the one hand, the importance of imagination in creative-innovative processes, and, on the other, the dialogical basis of division of labour. In this way, we propose that an integral reading of Smith's work is relevant, not only to reach a more complete understanding of the author's own economic ideas, but also to provide interesting suggestions for thinking about the dynamics of growth and development today.

* Recibido: 09/05/2021 - Aprobado: 16/06/2021 
Keywords: Adam Smith; Economic growth; Creativity; Innovation; Division of labour; Imagination; Language

\section{Introducción}

Como es bien sabido, para Adam Smith la división del trabajo resulta crucial en el proceso de crecimiento económico. Quizás sea difícil encontrar en otros autores a lo largo de la historia de la economía una descripción más minuciosa, a la vez que una defensa más encendida, acerca de los efectos y beneficios económicos de la especialización productiva que aquella del Libro I de la Riqueza de las Naciones, con sus antecedentes en las Lecciones sobre jurisprudencia, el Early Draft y los Fragmentos sobre la división del trabajo. Allende los propios reparos que Smith expresó sobre los posibles efectos negativos para una nación de una estructura productiva en la que la división del trabajo se llevara al extremo ${ }^{1}$, el énfasis puesto por Smith en el incremento de la productividad ocasionada por la división del trabajo llevó a que su concepción general sobre la dinámica del crecimiento económico se asociara casi exclusivamente con la especialización productiva del trabajo y la libre expansión los mercados competitivos mediante una interpretación, muy difundida en el ámbito de la economía, de la metáfora de la Mano Invisible.

El modelo de crecimiento económico smithiano se ha entendido, a partir de lo anterior, como opuesto, y hasta a veces obsoleto, en relación con otras aproximaciones más recientes en la historia del pensamiento económico, particularmente aquellas derivadas de las ideas de Schumpeter sobre la dinámica de la creatividad y la innovación a través de la noción de "destrucción creativa" (Nakamura, 2000; Andersson, 2009; Pol, 2013; Legros, Newman \& Proto, 2014; Gutiérrez Rojas \& Baumert, 2018; Ballor \& Claar, 2019).

En este artículo intentaremos mostrar otra perspectiva de la concepción smithiana del crecimiento económico centrada en dos aspectos fundamentales: por un lado, la importancia de la imaginación en los procesos creativo-innovativos, y, por otro, el basamento dialógico del proceso de división del trabajo. En cuanto al primer aspecto, nos remitiremos particularmente a dos de los textos incluidos dentro de los Ensayos filosóficos, la Historia de la astronomía y De la naturaleza de la imitación que tiene lugar en las llamadas artes imitativas, y a las 
Consideraciones sobre la primera formación de las lenguas. Allí señalaremos algunas ideas smithianas acerca de las relaciones entre sentimientos y creatividad haciendo foco en el papel central de la facultad de la imaginación, tanto en la creación de teorías explicativas, como de obras de arte y en la evolución del lenguaje. En cuanto al segundo aspecto, nos dirigiremos a la clásica exposición sobre la división del trabajo, al inicio de la Riqueza de las naciones, para revisar la descripción smithiana del proceso de innovación tecnológica a la luz del vínculo entre la propia división del trabajo y las capacidades lingüísticas del ser humano. Vínculo que manifiesta su importante significación al contraste con otros textos del corpus smithiano, como las Lecciones sobre jurisprudencia y la Teoría de los sentimientos morales. A partir de allí, se señalarán algunas de las consecuencias al momento de considerar el carácter intrínsecamente social y simpatético de la naturaleza humana en la interpretación del proceso de crecimiento económico.

Mediante este estudio proponemos, en última instancia, que una lectura integral de la obra smithiana no solamente importa para alcanzar una comprensión más acabada de las propias ideas económicas del autor, sino que incluso puede brindar interesantes sugerencias para pensar la dinámica del crecimiento y desarrollo, a diversos niveles, en la actualidad.

\section{Imaginación creativa en la Historia de la astronomía}

Si bien, como afirma Schliesser (2005), no se encuentra en la obra de Smith un tratamiento directo sobre la creatividad y la innovación en el ser humano, no obstante, en uno de los tres ensayos sobre Los principios que presiden y dirigen las investigaciones filosóficas, aquel conocido como la Historia de la astronomía, Smith llama la atención sobre ciertos aspectos y elementos del proceso de génesis, aceptación y reemplazo de teorías científico-filosóficas que sugieren ideas importantes al respecto.

En primer lugar, cabe recordar que Smith comienza ese opúsculo caracterizando tres sentimientos: el asombro causado por lo nuevo y singular, la sorpresa ante lo inesperado y la admiración producida por lo grandioso o hermoso, y en el párrafo final de la introducción expresa el objetivo fundamental del texto: "considerar en profundidad la naturaleza y causas de cada uno de estos sentimientos, cuya influencia es mucho más amplia de lo que un análisis descuidado podría hacernos imaginar" (Smith, 1998: 45). 
Al iniciar su indagación por el análisis de la sorpresa, Smith parece valerse de un principio humeano que puede separarse en dos proposiciones correlativas: 1) toda idea de un objeto produce una pasión o emoción correspondiente y 2) la intensidad de dicha pasión o emoción resulta directamente proporcional al grado de habitualidad de la mente en la concepción del objeto (Carrión, 2016). Al describir las consecuencias de la aparición de un objeto inesperado, con el consecuente incremento de la pasión correlativa, Smith afirma que en ciertas ocasiones

se descoyunta tan completamente el marco de la imaginación que nunca más puede retornar a su tono y compostura de antes, sino que se precipita al delirio o la locura habitual; y casi siempre produce una pérdida momentánea de la razón o de la atención a las cosas que requiere nuestra posición o nuestro deber (Smith, 1998: 45).

Es decir que existe una disposición habitual por la cual la imaginación prevé la aparición de determinados objetos, y lo propio de la sorpresa estriba en la reacción de la mente ante el modo de presentarse una emoción de manera repentina (Smith, 1998: 46).

Al ocuparse del asombro, Smith se vale de otro principio de fuerte resonancia humeana: "[e]s evidente que la mente se complace en observar las semejanzas que cabe descubrir entre objetos diferentes" (Smith, 1998: 49), y es precisamente gracias a la observación de tales semejanzas que la mente "procede a disponer y metodizar todas sus ideas, reduciéndolas a sus clases y variedades correspondientes" (Smith, 1998: 49). Pero cuando no nos resulta posible asociar un objeto a otro por semejanza sobreviene el asombro (Smith, 1998: 50). Por tanto, la brecha ante la aparición de ideas desconectadas es captada por la imaginación, lo que produce la emoción del asombro. Ahora bien, la imaginación no solamente capta esa separación, sino que lo desagradable de esta experiencia la mueve a construir algún tipo de vínculo entre tales ideas, de modo tal que la transición de una a otra sea más fácil y, por tanto, agradable.

Dicha permeabilidad de la imaginación ante las pasiones, que la mueve a "tender puentes" entre fenómenos separados, es uno de los tópicos centrales de la gnoseología smithiana. En efecto, esta tendencia a "llenar los vacíos intermedios" lleva a la imaginación a suponer hechos que conectan objetos, de manera tal que las hipótesis científico-filosóficas cumplen una función conectiva que ayuda a disminuir el asombro. En la descripción de 
este proceso conectivo Smith sostiene que existe una especie de inercia de la imaginación, moldeada por el hábito y la costumbre, en la transición entre ideas.

A partir de esas consideraciones sobre los procesos imaginativopasionales intrínsecos a la naturaleza humana se comprende la definición de la filosofía como "la ciencia de los principios conectivos de la naturaleza". Asimismo, Smith caracteriza su finalidad y procedimiento de la siguiente manera:

La filosofía, al exponer las cadenas invisibles que conectan todos esos objetos dislocados, pretende traer el orden a este caos de apariencias discordes y chirriantes, apaciguar el tumulto en la imaginación y restaurar en ella, cuando revisa los grandes cambios del universo, el tono de tranquilidad y compostura que le es al tiempo más grato de por sí y más conforme a su naturaleza. La filosofía, en consecuencia, puede ser considerada como una de las artes que se dirigen a la imaginación, cuya teoría e historia caen por ello propiamente dentro del ámbito de nuestra investigación (Smith, 1998: 57).

Interesa resaltar el enfoque práctico que Smith da a la filosofía: su importancia radica no tanto descubrir la verdad de las cosas, sino en tranquilizar a la imaginación construyendo teorías/puentes, mediante hipótesis/ladrillos que son, a su vez, supuestos/invisibles.

Este enfoque posibilita a Smith proponer un recorrido a través de la historia de la astronomía considerando la efectividad práctica para el incremento del placer/tranquilidad que las teorías brindan a la imaginación en cuanto hipótesis conectivas, de manera tal que la cuestión de la veracidad de la teoría queda relegada a un segundo plano:

Examinemos, entonces, los diferentes sistemas de la naturaleza que [...] han sido adoptados sucesivamente por las personas sabias e ingeniosas; $y$, sin considerar su absurdo o verosimilitud, su acuerdo o incompatibilidad con la verdad y la realidad, estudiémoslo sólo desde el enfoque particular que corresponde a nuestro tema, y limitémonos a investigar el grado en que cada uno de ellos estaba preparado para aliviar la imaginación, para transformar el teatro del mundo en un espectáculo más coherente y por ello más magnífico de lo que podría haber parecido en otro caso. Según lo hayan conseguido o no, habrán sistemáticamente logrado reputación y reconocimiento para sus autores o no; y se 
verá que ésta es la clave que mejor puede conducirnos a través de todos los laberintos de la historia filosófica; al tiempo sirve para confirmar lo que ha sucedido antes y arrojar luz sobre lo que puede venir después; y podemos observar en general que no hay sistema, por mejor fundamentado que haya estado en otros aspectos, que haya podido cosechar un crédito amplio en el mundo si sus principios conectivos no resultaban familiares a toda la humanidad (Smith, 1998: 57-58).

Este pasaje resulta clave para comprender tanto el objetivo del ensayo como la totalidad de la obra smithiana por varios motivos. En primer lugar, porque aquí el autor expresa la preeminencia de la acción de la imaginación por sobre la razón como factor explicativo de la construcción y sucesión de sistemas científico-filosóficos. En segundo lugar, porque señala las condiciones que estos sistemas deben cumplir para ser adoptados por la humanidad, cuales son, la unificación coherente de fenómenos y la familiaridad de los principios explicativos. En tercer lugar, destaca el elemento subjetivo presente en dicha aceptación y, con marcado realismo, Smith viene a decir que el reemplazo de un sistema no se debe a un mero proceso lógico-racional, sino que ocurre en gran medida gracias a los efectos emocionales generados por un nuevo escenario de conexiones entre ideas. Por último, afirma que este enfoque no solo será útil para analizar la historia de las ciencias sino también para prever las características de futuros sistemas, por tanto, podría colegirse que esta perspectiva adquiere en Smith un carácter programático para comprender sus propias teorías morales y económicas.

Este enfoque epistemológico smithiano le permite, a su vez, concebir a los sistemas explicativos como maquinarias imaginativas tendientes a devolver a la propia imaginación a su natural estado de tranquilidad y complacencia:

Los sistemas en muchos aspectos se asemejan a las máquinas. Una máquina es un sistema pequeño, creado para desarrollar y para conectar en la realidad los diferentes movimientos y efectos que el artesano necesita. Un sistema es una máquina imaginaria inventada para conectar en la mente los diversos movimientos y efectos que ya existen en la realidad. Las máquinas que son primero inventadas para efectuar cualquier marcha concreta son siempre las más complejas, y los artesanos posteriores generalmente descubren que con menos ruedas, con menos principios motrices que los empleados originalmente se pueden 
producir más fácilmente los mismos efectos. Análogamente, los primeros sistemas son siempre más complejos y por regla general se cree que es necesario una cadena o principio conectivo para enlazar todos los pares de fenómenos aparentemente desunidos; pero frecuentemente sucede que después se descubre que un solo gran principio conectivo es suficiente para ligar todos los fenómenos desacordes que tienen lugar en el conjunto de una especie de cosas (Smith, 1998: 75).

El caso más extraordinario de estas "máquinas imaginarias" en la historia de la astronomía, para Smith, lo representa el sistema newtoniano. Esto es así porque cumple más acabadamente con los distintos criterios epistémicos que el escocés introduce a lo largo de su narración sobre la sucesión de sistemas astronómicos, entre los que se cuentan: el principio de parsimonia, la familiaridad del principio explicativo (la gravedad), su corroboración empírica, su capacidad explicativa y su coherencia interna. No obstante, cabe señalar que existe un criterio muy importante para comprender la aceptación generalizada de un sistema explicativo o, dicho de otra forma, una "maquina imaginaria", cual es la belleza del sistema. La importancia de la cuestión estética dentro del pensamiento smithiano amerita una digresión antes de abordar la cuestión de la innovación y la división del trabajo.

\section{Creaciones artísticas, valoración estética y lenguaje}

Según lo dicho anteriormente, la belleza de un sistema explicativo figura entre las condiciones de su aceptación, pero en la Historia de la astronomía no se da una definición de belleza. Para encontrarla es necesario remitirse al ensayo titulado De la naturaleza de la imitación que tiene lugar en las llamadas artes imitativas.

En dicha obra Smith sostiene que suele denominarse belleza a cierta semejanza basada en la simetría de proporción dentro una disposición dada de cosas (Smith, 1998: 173). No obstante, esta definición vale para la calificación de los objetos tal como se dan en la naturaleza. Las obras de arte, en cuanto creaciones humanas, deben cumplir con otra característica para ser consideradas bellas: deben unificar mediante ese tipo de semejanza objetos que en la naturaleza presentan grandes diferencias (Smith, 1998: 181). El mérito de las artes imitativas radica, pues, en su capacidad para conectar dos elementos per se diferentes (el representado y el representante) mediante un proceso determinado. Dice Smith: 
El placer apropiado que derivamos de esas dos artes imitativas [la estatuaria y la pintura], lejos de ser el efecto del engaño, es totalmente incompatible con él. Dicho placer se basa por entero en nuestra maravilla al contemplar un objeto de una clase que representa tan bien a otro objeto de una clase muy diferente, y en nuestra admiración hacia el arte que tan felizmente supera la disparidad que la naturaleza ha establecido entre ellos. Las maravillas de la estatuaria y la pintura nos parecen fenómenos prodigiosos, que difieren de los fenómenos formidables de la naturaleza en que transportan con ellos, por así decirlo, su propia explicación, y demuestran, incluso a ojos vistas, la forma y manera en que han sido producidos. [...] La placentera admiración de la ignorancia viene acompañada de la aún más placentera satisfacción de la ciencia. El efecto nos maravilla y asombra; y nos gusta y satisface el verificar que podemos comprender en algún grado cómo se produce tan prodigioso efecto (Smith, 1998: 182) ${ }^{2}$.

Así como el fin de la investigación científico-filosófica consistía en devolver a la imaginación su estado de reposo natural -alterado por la sorpresa y el asombro- mediante un sistema que conectara fenómenos aparentemente inconexos, las artes imitativas consiguen su objetivo cuando generan en el ser humano los sentimientos placenteros de admiración y asombro mediante la producción de una nueva relación de semejanza entre elementos dispares a través de procedimientos identificables.

Ahora bien, si tanto las artes como la filosofía se dirigen a la imaginación, los criterios estéticos y epistémicos no pueden separarse estrictamente. En efecto, la captación de semejanzas y la construcción de puentes entre objetos diversos, tendientes a lograr una unidad sistemática entre fenómenos, están en la base tanto del origen de las ciencias como de las artes, puesto que ambas persiguen como fin último el placer y tranquilidad de la imaginación. A propósito, es significativa la comparación que establece Smith entre un concierto musical y un sistema científicofilosófico:

Al contemplar esa inmensa multiplicidad de sonidos gratos y melodiosos, arreglados y recopilados, tanto en su coincidencia como en su sucesión, en un sistema tan completo y regular, la mente en realidad disfruta no sólo de un muy intenso placer sensual sino también de un elevado placer intelectual, no disímil del que deriva de la contemplación de un gran sistema en cualquier otra ciencia (Smith, 1998: 202) ${ }^{3}$. 
Asimismo, el elemento estético cumple una importante función en la explicación acerca del origen y evolución de los lenguajes, según la exposición de las Consideraciones sobre la primera formación de las lenguas. En este trabajo, Smith trata de explicar el modo en que se generaron las lenguas a partir de la creación de los diferentes tipos de palabras que las conforman, hasta hacer una crítica a los idiomas romances preposicionales en relación con los clásicos desinenciales ${ }^{4}$. El hilo conductor del ensayo radica en la idea según la cual las palabras y conceptos más abstractos y generales, por tanto, más distantes para las capacidades intelectuales humanas, se forman a partir de objetos individuales, más concretos y cercanos, de la vida cotidiana. Al mismo tiempo, se trata de demostrar que la evolución en la articulación de los lenguajes se vincula estrechamente con el placer generado por cierta semejanza y uniformidad en la sonoridad de las palabras. En efecto, al dar cuenta de la creación de los adjetivos en las lenguas desinenciales, afirma Smith:

Los hombres naturalmente les daban las mismas terminaciones que a los sustantivos a los que se le aplicaban primeramente, y a partir de ese amor a la similitud de sonido, de ese deleite por la repetición de las mismas sílabas, que es el fundamento de la analogía en todas las lenguas, tendían a variar la terminación del mismo adjetivo (Smith, 2017: 108).

La semejanza, entonces, no solo es relevante para explicar la formación de términos generales mediante la asociación de objetos individuales, sino que además ayuda a entender las relaciones entre dichos términos según su expresión sonora. Es decir que las relaciones de semejanza no solo son importantes a nivel de la forma sino también de la materia del lenguaje. Y esto precisamente porque Smith reconoce una especie de propensión natural hacia la semejanza, la cual se vincula con el placer por ella generado; de aquí las nociones de "amor y deleite por la similitud de sonido". El gusto por la captación de semejanzas tanto a nivel de ideas/imágenes como a nivel sonoro explica, pues, el desarrollo de las lenguas, siendo esta una característica propia de la naturaleza humana.

$\mathrm{Al}$ reflexionar sobre los elementos que configuran el devenir de las lenguas, Smith llega a la enunciación de una regla general:

puede establecerse como máxima que cuanto más simple sea una lengua en su composición, más compleja debe ser en sus declinaciones y conjugaciones, y, por el contrario, cuanto más 
simple en sus declinaciones y sus conjugaciones, más compleja será en su composición (Smith, 2017: 125).

Es significativo que Smith utilice en este punto una vez más la analogía mecánica, comparando el desarrollo de las lenguas con el perfeccionamiento de las máquinas. En un primer momento estas pueden resultar muy complejas y requieren de muchos elementos para concretar sus movimientos. Pero con el tiempo, los técnicos descubren diversos modos para economizar recursos logrando la misma cantidad de movimientos, de manera tal que la máquina se vuelve más simple en su conjunto (Smith, 2017: 127).

No obstante, la analogía mecánica respecto de los lenguajes no es estricta, lo que se relaciona directamente con el criterio estético. Mientras que el proceso de economía y simplificación en las máquinas es siempre progresivo, pues tiende a perfeccionarlas, para Smith este mismo proceso resulta regresivo para muchos propósitos del lenguaje, ante lo cual brinda tres argumentos. En primer lugar, sostiene que esa simplificación vuelve a las lenguas más perifrásticas, ya que necesitan relativamente más palabras para expresar las mismas cosas. Este exceso en la incorporación de términos, según Smith, disminuye la elocuencia de las lenguas modernas en comparación con las clásicas, dado que "la belleza de una expresión depende en su concisión" (Smith, 2017: 128). En segundo lugar, pero estrictamente relacionado con lo anterior, afirma que la simplificación hace a los lenguajes menos agradables al oído, al perderse la variedad de las terminaciones propias de las lenguas desinenciales. Por último, las lenguas preposicionales presentan menos posibilidades para ordenar los términos, particularmente en las composiciones poéticas, en vistas a lograr el mayor efecto estético según sus sonidos.

Teniendo en cuenta las anteriores consideraciones sobre las relaciones entre la valoración estética, la creación artística y la evolución de las lenguas en el pensamiento de Smith, a continuación, se propondrá una interpretación de su análisis de la división del trabajo y la incorporación de innovaciones tecnológicas, en tanto fenómenos intrínsecamente sociales, sobre la base de la propia concepción smithiana imaginativo-pasional de la naturaleza humana. 


\section{División del trabajo, innovación y la naturaleza dialógico- simpatética del ser humano}

En la introducción de la Riqueza de las naciones, Smith define la riqueza nacional como la proporción entre el producto anual y la cantidad de personas que lo consumen. Esta proporción depende especialmente de una circunstancia: "la aptitud, destreza y sensatez con que generalmente se ejercita el trabajo" (Smith, 1999: 3). Ahora bien, los niveles de "aptitud, destreza y sensatez" del trabajo dependen, a su vez, del desarrollo que tenga en la nación la división del trabajo. De aquí que las condiciones, causas y consecuencias de la división del trabajo se conviertan en algunas de las temáticas más importantes de la obra.

El principal efecto positivo que genera la división del trabajo consiste en el aumento de la productividad, ilustrado mediante el famosísimo ejemplo de la fábrica de alfileres (Smith, 1999: 8-9)5. Tres son las causas que producen tal incremento:

1. La mayor destreza que cada obrero adquiere al dedicarse a una única tarea,

2. El ahorro de tiempo por no tener que cambiar de ocupación,

3. El incentivo para la invención de maquinarias que faciliten los trabajos (Smith, 1999: 10-12).

Resulta importante notar que para Smith el propio proceso de innovación tecnológica depende de la división del trabajo. A propósito, afirma: "[e]l hombre adquiere una mayor aptitud para descubrir los métodos más idóneos y expeditos, a fin de alcanzar un propósito, cuando tiene puesta toda su atención en un objeto, que no cuando se distrae en una gran variedad de cosas" (Smith, 1999: 12). De esta manera, el ejercicio de enfocarse en una tarea que produce la división del trabajo aguza, para Smith, el ingenio de los trabajadores para desarrollar maquinarias que faciliten sus tareas. En este sentido, resulta igualmente significativa la afirmación smithiana según la cual estos primeros innovadores no fueron grandes filósofos o científicos, sino, precisamente, los propios trabajadores que debían que esforzarse en realizar la tarea y, de esta manera, trataron de lograr una solución inmediata a un problema práctico: 
Una gran parte de las máquinas empleadas en esas manufacturas, en las cuales se halla muy subdividido el trabajo, fueron al principio invento de artesanos comunes, pues hallándose ocupado cada uno de ellos en una operación sencilla, toda su imaginación se concentraba en la búsqueda de métodos rápidos y fáciles para ejecutarla (Smith, 1999: 12-13).

Después de señalar este tipo de innovación propia de los trabajadores, Smith reconoce que no solamente estos han sido capaces de producir adelantos tecnológicos, sino también aquellas personas que, si bien no operan las maquinas, están habituadas a fabricarlas y, por último, "los llamados filósofos y hombres de especulación, cuya actividad no consiste en hacer cosa alguna sino observarlas todas y, por esta razón, son a veces capaces de combinar o coordinar las propiedades de los objetos más dispares" (Smith, 1999: 13). En este pasaje Smith parece reconocer distintos niveles de innovaciones, desde las más simples, concretas y prácticas a las más complejas, abstractas y teóricas, y dentro de estas últimas, aparece nuevamente el tópico de la conexión de objetos dispares que, como se vio, es la clave según la cual Smith entiende tanto la génesis y evolución de la ciencia como del arte.

En el último párrafo del primer capítulo del Libro I de la Riqueza, Smith describe con gran fuerza retórica la enorme cantidad de personas que intervienen en el proceso de intercambio que permiten, en un país civilizado, que hasta los trabajadores más humildes tengan a disposición los productos que requieren para la vida:

[S]i nos detenemos, repito, a examinar todas estas cosas y a considerar la variedad de trabajos que se emplean en cualquiera de ellos, entonces nos daremos cuenta de que sin la asistencia y cooperación de millares de seres humanos, la persona más humilde en un país civilizado no podría disponer de aquellas cosas que se consideran las más indispensables y necesarias (Smith, 1999: 15).

Esta afirmación pone en el centro de la cuestión de la división del trabajo la idea de interacción social del proceso de intercambio, es decir, la naturaleza intrínsecamente social de la actividad económica. Pero una vez señalado esto, Smith pasa a preguntarse, en el segundo capítulo, por la causa de la división del trabajo. 
Allí se apresura a señalar que no corresponde a la sabiduría humana ser causa eficiente de la división del trabajo, ya que los individuos no pueden prever los efectos positivos de su acción individual para el incremento de la riqueza de la nación. Por tanto, la respuesta es la siguiente: "[e]s la consecuencia gradual, necesaria aunque lenta, de una cierta propensión de la naturaleza humana que no aspira a una utilidad tan grande: la propensión a permutar, cambiar y negociar una cosa por otra" (Smith, 1999: 16).

Inmediatamente después de explicitar el origen de la división del trabajo, Smith aclara que no se ocupará en ese texto del problema relativo al estatuto de dicha propensión a trocar, es decir, si se trata propiamente de un principio innato del ser humano o, más bien, un efecto de sus facultades discursivas. No obstante, en las Lecturas sobre jurisprudencia había dicho:

Si investigáramos sobre el principio de la mente humana sobre el que se basa esta disposición al cambio, sería claramente la inclinación natural, que cada uno tiene, a persuadir. La oferta de un chelín, que para nosotros parece que tiene un sentido tan claro y simple, es ofrecer un argumento, en realidad, para persuadir a alguien de hacer tal o cual cosa porque le interesa. Los hombres siempre se esfuerzan en persuadir a los otros de que sean de su opinión, incluso cuando el asunto no les afecta (Smith, 1996: 400).

En este pasaje la disposición a trocar se entiende como un efecto de una propensión anterior, la inclinación a persuadir. De esta manera, todo intercambio comercial estaría guiado, en última instancia, por un deseo natural de hacer que los demás acepten el punto de vista personal, lo que remite a algunas importantes consideraciones sobre la estructuración social y la acción humana en Teoría de los sentimientos morales (Montes, 2019).

Dado que el ser humano es naturalmente social y simpatético, su propia felicidad dependerá inevitablemente del tipo de vínculo que establezca con los demás. Como es bien sabido, precisamente esta cuestión tema constituye el punto de partida de la Teoría, en clara oposición a los planteos egoístas-racionalistas de posturas como la hobbesianamandevilleana. A propósito, Smith no solamente afirma que a los individuos nos importa la felicidad de los demás, sino que un aspecto de la propia felicidad consiste en ser considerados por los demás y tener conciencia de ello (Smith, 2004: 106). Esto significa, en un primer momento, el mero hecho de ser tenido en cuenta por las otras personas y, posteriormente, la 
búsqueda de la emoción derivada de la aprobación de sus acciones y motivos: "¿Qué mayor felicidad hay que la de ser amado y saber que lo merecemos? ¿Qué mayor desgracia que la de ser odiado y saber que lo merecemos?" (Smith, 2004: 225).

De acuerdo con lo anterior, la comunicación dialógica y la cooperación en términos socio-económicos entre los seres humanos están intrínsecamente relacionadas para Smith, dada su concepción imaginativopasional de la naturaleza humana. En este sentido, la persecución del interés individual está siempre mediada por los vínculos simpatéticos, más o menos intensos según grados de proximidad, establecidos entre los miembros de una sociedad. Esto implica, a su vez, que los procesos de creación e innovación, tanto a nivel teórico (hipótesis explicativas) como práctico (obras de arte y maquinarias), que ayudan a entender el progreso de las naciones, deben entenderse y promoverse a partir de dicho basamento antropológico. La interpretación de la problemática del crecimiento económico en Smith, por tanto, requiere un abordaje complejo y multidimensional que, por una parte, aboga por la recuperación del pensamiento del autor desde una visión de conjunto, y, por otra, adquiere una especial relevancia para analizar y comprender el devenir de las sociedades y economías contemporáneas.

\section{A modo de conclusión}

A través de este artículo intentamos mostrar que una lectura integral de la obra de Adam Smith no solo resulta importante para reinterpretar sus ideas en el ámbito de la economía, sino también para aportar sugerencias relevantes a cuestiones actuales relativas a los procesos de crecimiento y desarrollo. Argumentamos que la concepción generalizada sobre crecimiento económico en la Riqueza de las naciones, mediante la división del trabajo y la expansión de mercados competitivos, conlleva una complejidad poco reconocida al vincular la teorización económica de Smith con sus ideas epistemológicas, estéticas, lingüísticas y ético-sociales.

Desde dicho amplio marco de compresión se vio que la génesis y aceptación de explicaciones científico-filosóficas depende de la relación entre, por una parte, los sentimientos de sorpresa, asombro y admiración, y, por otra, la acción de la imaginación, dada su capacidad creativa para establecer conexiones entre fenómenos que aparecen como distantes y desconectados. Esta aproximación epistemológica implica entender las hipótesis explicativas a la manera de artefactos creados principalmente para 
tranquilizar a la propia imaginación, de manera tal que, en tanto productos de elaboración humana, el elemento estético de los sistemas explicativos resulta valorado para comprender no solo la creación de una teoría sino también su aceptación por la sociedad. Así, el enfoque smithiano torna borrosas las fronteras entre los criterios epistémicos, estéticos y técnicos, para comprender las teorías científico-filosóficas, las obras de arte y las máquinas que permiten incrementar la productividad en términos económicos.

Teniendo en cuenta lo anterior, se insistió en el proceso de incorporación de innovaciones tecnológicas a partir del fenómeno de la división del trabajo, señalando el hecho de que, para Smith, personas imbricadas en diversas esferas del mundo laboral, desde los ámbitos más prácticos y concretos hasta los más teóricos y abstractos pueden introducir distintos tipos de innovaciones. Pero principalmente se hizo hincapié en la observación smithiana acerca del fundamento lingüístico de la división del trabajo, ya que esto implica el reconocimiento de la naturaleza intrínsecamente social y simpatética de la naturaleza humana, cuestión que Smith desarrollara particularmente en la Teoría de los sentimientos morales, y que encuentra en la facultad del habla y la persuasión un nexo comunicante, no solamente entre los dos grandes textos publicados por el escocés, sino entre toda su obra.

Entendemos que esta interpretación de la concepción smithiana de la dinámica del crecimiento económico resulta importante en la actualidad puesto que plantea la integración de aristas esenciales de la acción humana, tanto individual como social, cuyas relaciones con los procesos de desarrollo a nivel local, regional, nacional y transnacional, se están analizando en profundidad desde hace no mucho tiempo. Entre estos, cabe destacar, en primer lugar, la relevancia del factor imaginativo y emocional en su impacto sobre la creatividad y la innovación, así como también de la dimensión comunicacional, en comprensión de la evolución de las economías (Loasby, 2002, 2014; Wight, 2009). En segundo lugar, la importancia de la apreciación y formación estética-artística en los procesos de desarrollo, tanto a partir del estímulo de la propia creatividad como de los vínculos simpatéticos que se generan y refuerzan en los espacios comunes destinados a la cultura (Sacchetti, Sacchetti \& Sugden, 2009). En tercer lugar, la necesidad de una mayor y mejor comunicación y cooperación entre agentes innovadores, a diferentes niveles y de diferentes ámbitos, es una idea que han sido puesta en el centro de las discusiones actuales sobre las economías 
basadas en el conocimiento a partir de la noción de Innovación abierta (Chesbrough, 2003; 2020). Por último, el anclaje territorial de los procesos de desarrollo local-regional, implícita en la noción smithiana de la estructuración social a partir de vínculos simpatéticos entre individuos condicionados por su espacio vital, es destacada en la actualidad desde el denominado Enfoque del desarrollo territorial (Alburquerque, 2013; Costamagna, 2015). De este modo, la perspectiva filosófico-antropológica y socio-económica smithiana muestra su potencial, todavía hoy, como un marco de referencia abierto, complejo y dinámico para poner en diálogo e integrar estos y otros enfoques analíticos contemporáneos y avanzar, así, en la comprensión teórica y la concreción práctica de procesos de crecimiento y desarrollo.

\section{Referencias Bibliográficas}

Alburquerque, F. (2013). Economía del Desarrollo y Desarrollo Territorial. Recuperado de: http://www.conectadel.org/wpcontent/uploads/downloads/2015/03/E\%C2\%AADesarrollo-yDesarrollo-Territorial-3.01.pdf

Andersson, Å. E. (2009). "Economics of Creativity". En Karlsson, C. et al. (Eds.). New Directions in Regional Economic Development (pp. 7995). Berlin: Springer.

Ballor, J. \& Claar, V. (2019). "Creativity, innovation, and the historicity of entrepreneurship". Journal of Entrepreneurship and Public Policy, $8(4), 513-522$.

Carrión, G. (2016). "Estructura imaginativo-pasional de la naturaleza humana y economía en David Hume". Filosofía de la Economía, Vol. 5, 5-26.

Carrión, G. (ed.) (2017). Escritos preliminares de la Riqueza de las Naciones y consideraciones sobre la primera formación de las lenguas. Madrid: Biblioteca Nueva.

Chesbrough, H. W. (2003). Open Innovation: The new imperative for creating and profiting from technology. Boston: Harvard Business School Press.

Chesbrough, H. W. (2020). Open Innovation Results: Going Beyond the Hype and Getting Down to Bussines. New York: Oxford University Press.

Costamagna, P. (2015). Política y formación en el desarrollo territorial. Aportes al enfoque pedagógico y a la investigación. Bilbao: Orkestra-Instituto Vasco de Competitividad.

Gutiérrez Rojas, C. \& Baumert, T. (2018). "Smith, Schumpeter y el estudio de los sistemas de innovación”. Economía y Política, 5(1), 93-111. 
Legros, P., Newman, A. \& Proto, E. (2014). "Smithian Growth Through Creative Organization". The Review of Economics and Statistics, 96(5), 796-811.

Loasby, B. J. (2002). "The evolution of knowledge: beyond the biological model". Research Policy, 31(8-9), 1227-1239.

Loasby, B. J. (2014). "The Evolution of Knowledge and Knowledge of Evolution". Jahrbücher $f$. Nationalökonomie u. Statistik, Vol. 234/2+3, 142-157.

Montes, L. (2019). "Adam Smith's foundational idea of sympathetic persuasion". Cambridge Journal of Economics, 43(1), 1-15.

Nakamura, L. (2000). "Economics and the New Economy: The Invisible Hand Meets Creative Destruction". Business Review (Federal Reserve Bank of Philadelphia), July-August, 15-30.

Pol, E. (2013). "Reconciling the Invisible Hand and innovation". Economics of Innovation and New Technology, 22(5), 431-446.

Rosenberg, N. (1965). "Adam Smith on the Division of Labour: Two Views or One?”. Economica, 32(126), 127-139.

Sacchetti, F., Sacchetti, S. \& Sugden, R. (2009). "Creativity and socioeconomic development: space for the interests of publics". International Review of Applied Economics, 23(6), 653-672.

Schliesser, E. (2005). "Wonder in the Face of Scientific Revolutions: Adam Smith on Newton's 'Proof' of Copernicanism”. British Journal for the History of Philosophy, 13(4), 697-732.

Smith, A. (1995). Lecciones sobre jurisprudencia (Curso 1762-3). Granada: Comares.

Smith, A. (1996). Lecciones sobre jurisprudencia [Reporte de 1766]. Madrid: BOE/CEC.

Smith, A. (1998). Ensayos filosóficos. Madrid: Pirámide.

Smith, A. (1999). Investigación sobre la naturaleza y causas de la riqueza de las naciones. México: FCE.

Smith, A. (2004). Teoría de los sentimientos morales. Madrid: Alianza.

Smith, A. (2017). "Consideraciones sobre la primera formación de las lenguas y el diferente genio de las lenguas originales y compuestas". En Carrión, G. (ed.). Escritos preliminares de la Riqueza de las naciones y consideraciones sobre la primera formación de las lenguas. Madrid: Biblioteca Nueva.

Wight, J. (2009). "Adam Smith on Instincts, Affection, and Informal Learning: Proximate Mechanisms in Multilevel Selection”. Review of Social Economy, 67(1), 95-113.

\footnotetext{
${ }^{1}$ Sobre la famosa advertencia del Libro V de la Riqueza y su relación con lo expuesto en la primera parte de la obra puede verse el ya clásico artículo de Rosenberg (1965).

2 Más adelante incluye entre las condiciones de las artes imitativas la velocidad con que permiten pasar el objeto imitador al imitado: "En las artes imitativas, aunque no es en absoluto necesario que el objeto imitador se parezca al imitado tan exactamente que se confunda con este, es sin embargo necesario que ambos se asemejen al menos de modo que uno sugiera rápidamente al otro" (Smith, 1998: 193).
} 
3 Resulta interesante notar que en el contexto de discusión acerca de los efectos pasionales ejercidos por la música, Smith describe lo que ya en la Historia de la astronomía había denominado "estado natural de la mente" como una situación de equilibrio entre un estado jovial y otro de aflicción, es decir, como un estado de tranquilidad; que no se caracteriza solamente por el placer que genera sino también por un modo particular de transición entre ideas (Smith, 1998: 194).

4 Sobre la importancia de este texto dentro del corpus smithiano remitimos a nuestro "Estudio Introductorio" (Carrión, 2017).

5 Este ejemplo ya había sido utilizado en Smith (1995: 388-389, 1996: 136-137) y en el "Borrador" de la Riqueza de las naciones (Carrión, 2017: 137-138). 